\title{
The Blood Sugar Content of the Heat=punctured Rabbit.
}

\author{
$\mathrm{Br}$ \\ SACHIKADO MORITA and MASARU NAITO. \\ (森田幸門) (內 藤勝) \\ (From the Physiological Laboratory of Prof. Y. Satake, \\ Tōōku Imperial University, Sendai.)
}

This problem was treated first by P. F. Richter. ${ }^{1)}$ He gave a certain volume of a solution of glucose, saccharose or laevulose to the heat-punctured rabbits. Glycosuria occurred in exceptional cases. But a diminution of the liver glycogen content and a slight increase of the blood sugar content in eighteen hours after the puncture were discovered. H. Senator ${ }^{2}$ observed also hyperglycaemia on rabbits twenty hours after the heat-puncture, though slightly.

The recent studies of Yoshi. $\mathrm{Kuno} o^{3)}$ and Ko. $\mathrm{Na}$ ito ${ }^{4)}$ are in opposition to them. Kuno detected hyperglycaemia, of which the maximum was about three hours after the puncture on the successfully heatpunctured rabbits, but also in the unsuccessful cases. So, he concluded that the higher body temperature is not the cause of hyperglycaemia. Ko. Naito could not detect any hyperglycaemia on rabbits in two or four hours after the puncture.

The former experimentors estimated the blood sugar first in 18-20 hours after the heat-puncture, whereas the latter in only a few hours. 'Therefore, those two series of experiments can hardly be compared.

1) P.F.Richter, Fortschr. d. Med., 16 (1896), 321.

2) H. Senato r, Ztsehr. f. kl. Med., 67 (1907), 258.

3) Yoshi. Kuno, Tokyo-Igakkwaj-Zasshi, 28 (1914), 1423. (Jap.) and Mitteil. med Fak. Univers. Tokyo, 22 (1919), 175.

4) Ko. Na it o, Tohoku-Igaku-Zasshi, 4 (1919), 128. (Jap.) 
But, Yoshi. Kuno observed also lately the blood sugar content in 2329 hours after the heat-puncture on three rabbits, and always folnd hypergiycaemia, though not so marked (The excess was $0.02-0.07 \%$. Tab. IV in his second paper. Kuno himself did not refer to this event in his text). This seems to coincide with the findings of Senator and his co-worker.

This divergency of the earlier experimental results and the advancement of method of blood sugar estimation have lead us to treat this problem anew. .

Only mature male rabbits of good nutrition were used. The food and treatment of animals, blood drawing, estimation of the blood and urine sugar, measurement of the body temperature etc. were made as usual in our Laboratory.

The heat-puncture was carried out as in the description of Ed. Arohnson and J. Sachs.')

On the day previous to the principal experiment, the control experiment was carried out. The skull bone was perforated and the dura mater was cut open, but without being punctured, the skin over it was closed by means of artery forceps. On the next day the wound was opened and the puncture was performed.

'The time relation of drawing of blood samples, collection of urine etc. was just as in the case of the cold-puncture experiment by one of us. ${ }^{2)}$

The rabbits took food (tofulara) as usual on the day of the control experiment, but on the day of the puncture and following days they ate somewhat less, became thinner day after day and finally died in one or two months after the puncture.

Our experiments were performed in March, April, September and October.

All the experiments are shown in a table and some of them shall be illustrated in detail.

1) Ed. Arohnson and J. Sachs, Arch. f. ges. Physiol., 37 (1885), 235.

2) Sachi. Morita, this Journ., 2 (1921), 410. 


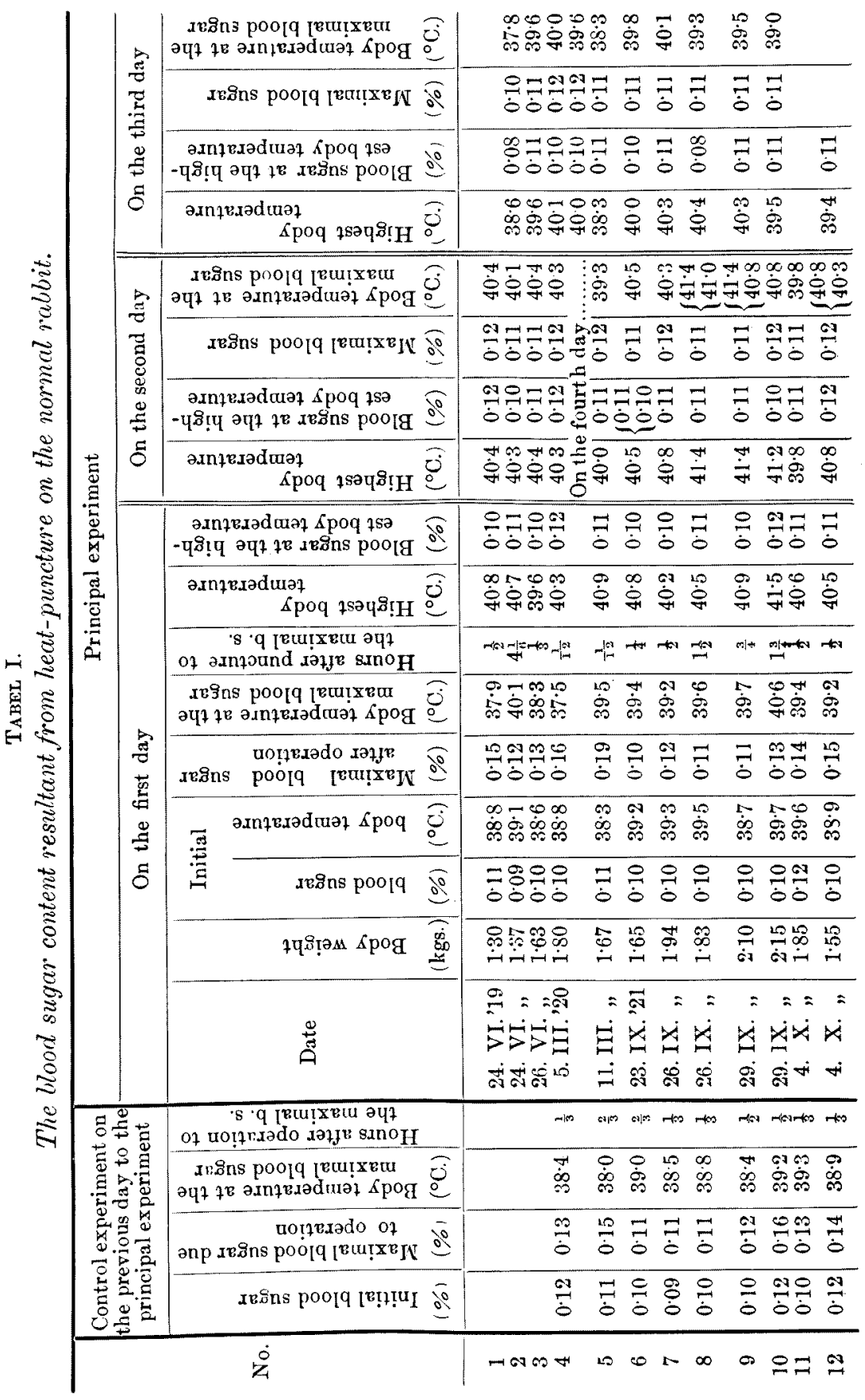


Experimext V.

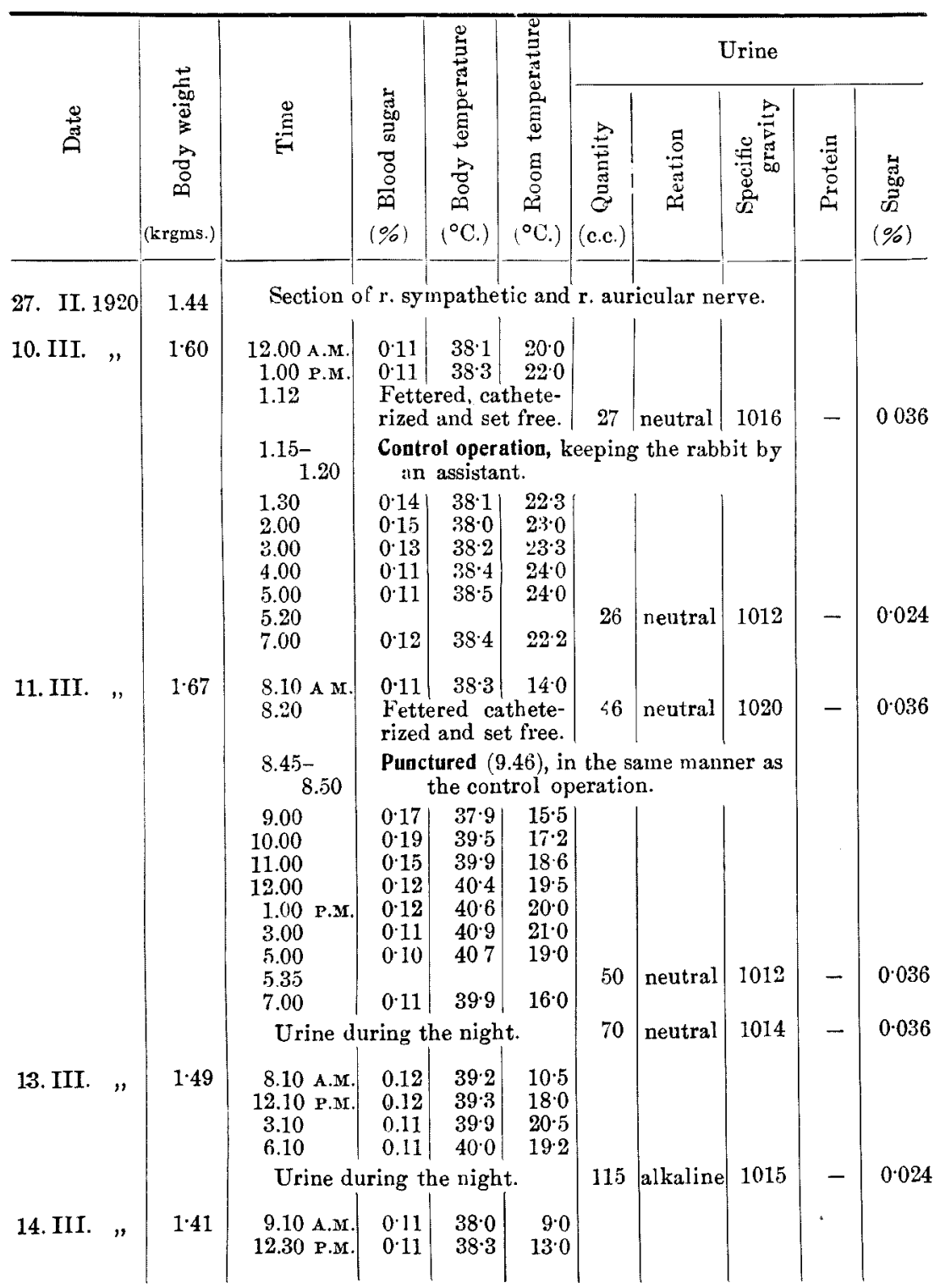


Experiment ViI.

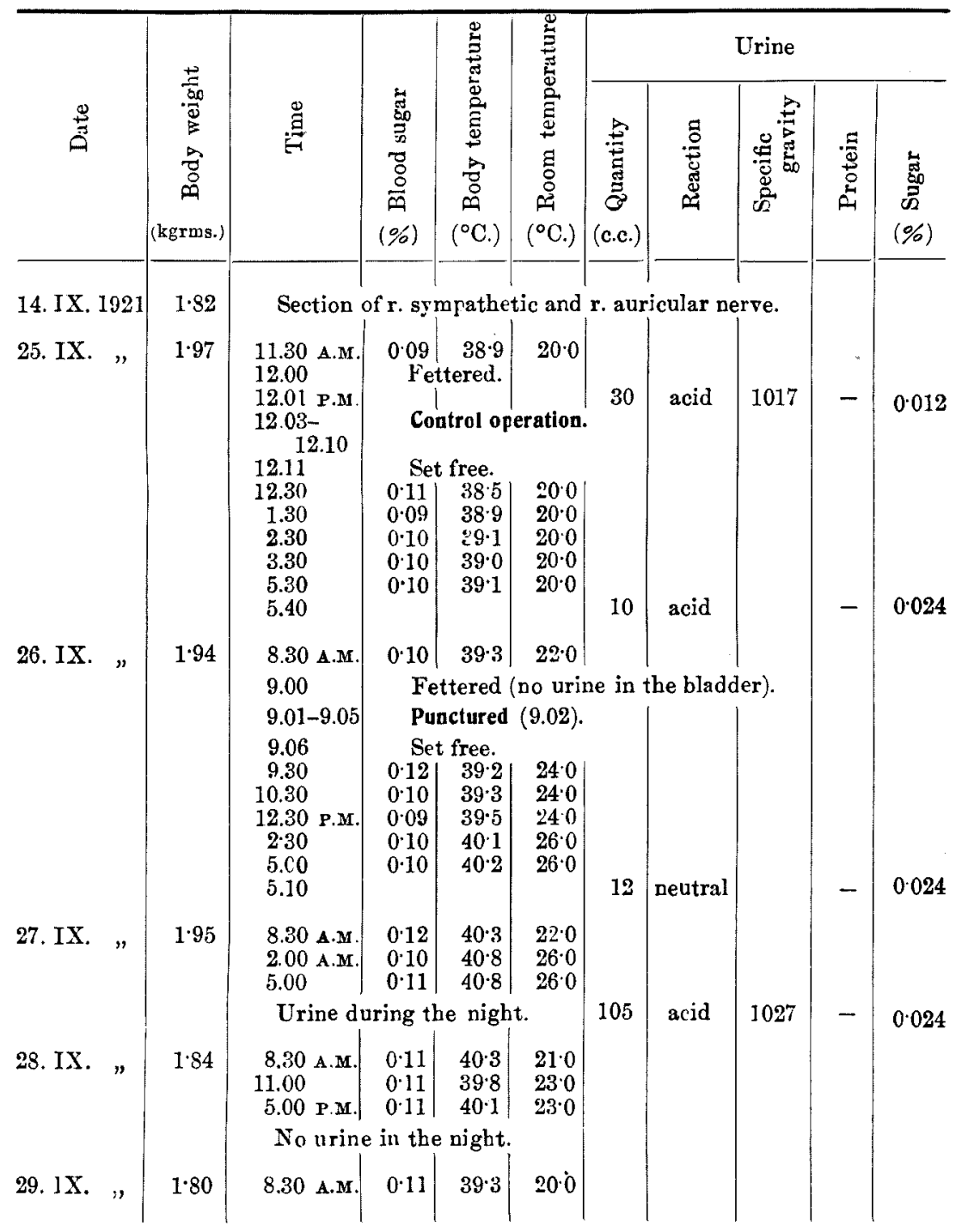

The body temperature rose gradually after the puncture and it reached over $40^{\circ} \mathrm{C}$. in $2-8$ hours after the puncture. The body temperature over $40^{\circ} \mathrm{C}$. continued on the day of the puncture and the next day in the majority of cases. The maximum was $40 \cdot 3-41.5^{\circ} \mathrm{C}$ in our cases.

The operation hyperglycaemia usually occurred on the control as 
EXPERIMENT X.

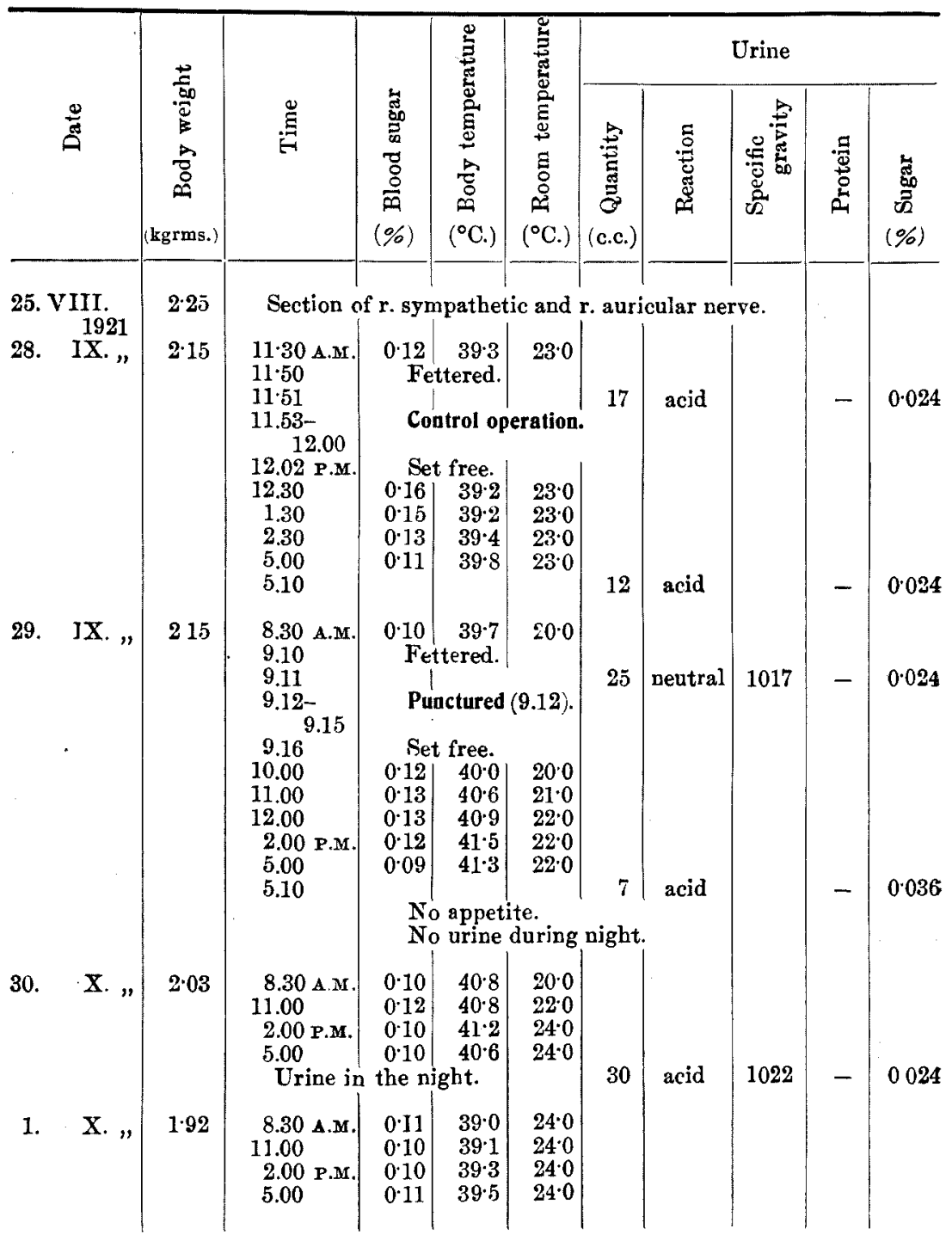

well as principal experiments. Otherwise, on the day of the puncture and the next day, where the body temperature was usually over $40^{\circ} \mathrm{C}$., there was no indication of hyperglycaemia and glycosuria. A small variation of the blood sugar content must be looked upon as physiolo- 
gical, judging from the experiments of A. Th. B. Jacobsen, ${ }^{1)}$ M. Kageyama,, T. Suzuki, ${ }^{3)}$ O. Asakawa ${ }^{4)}$ and I. Fujii ${ }^{5)}$ who estimated the daily variation of the blood sugar content in the normal animal with the micromethod of I. Bang, and our own estimations (Tab. II).

TABLE II.

The daily variation of the blood sugar of the normal rabbit.

Rabbit No. 1.

\begin{tabular}{|c|c|c|c|c|c|c|c|c|c|}
\hline 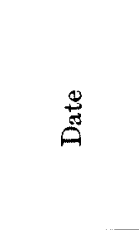 & 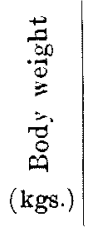 & 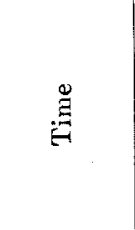 & 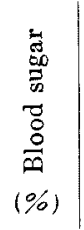 & 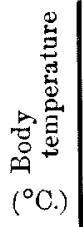 & 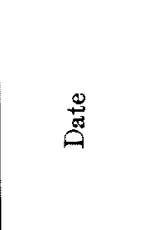 & 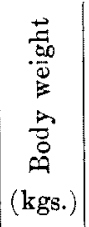 & $\stackrel{\Xi}{\Xi}$ & 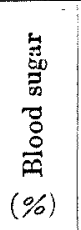 & 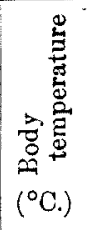 \\
\hline 9. IX '19 & $1 \cdot 95$ & $\begin{array}{r}11.00 \text { A.M. } \\
2.00 \text { P.M. } \\
5.00\end{array}$ & $\begin{array}{l}0.08 \\
0.09 \\
0.09\end{array}$ & $\begin{array}{l}38 \cdot 9 \\
39 \cdot 1 \\
39 \cdot 6\end{array}$ & 23. IX.' 19 & 1.82 & $\begin{array}{c}8.30 \text { A.M. } \\
11.30 \\
2.30 \text { P.M. } \\
5.00\end{array}$ & $\begin{array}{l}0 \cdot 11 \\
0 \cdot 10 \\
0.10 \\
0.09\end{array}$ & $\begin{array}{l}38 \cdot 9 \\
38 \cdot 9 \\
39 \cdot 3 \\
39 \cdot 6\end{array}$ \\
\hline 10. IX. " & 1.90 & $\begin{array}{l}8.00 \text { A.M. } \\
11.00 \\
2.00 \text { P.M. } \\
5.00\end{array}$ & $\begin{array}{l}0.09 \\
0.09 \\
0.08 \\
0.09\end{array}$ & $\begin{array}{l}38 \cdot 4 \\
38 \cdot 4 \\
38 \cdot 8 \\
39 \cdot 7\end{array}$ & 24. IX. , & 1.92 & $\begin{array}{c}8.30 \text { A.M. } \\
11.30 \\
2.30 \text { P.M. } \\
5.30\end{array}$ & $\begin{array}{l}0.11 \\
0.11 \\
0.11 \\
0.11\end{array}$ & $\begin{array}{l}39 \cdot 2 \\
39 \cdot 1 \\
39 \cdot 1 \\
39 \cdot 6\end{array}$ \\
\hline 11. IX. " & 1.93 & $\begin{array}{l}9.00 \text { A.M. } \\
12.00 \\
3.00 \text { P.M. } \\
6.00\end{array}$ & $\begin{array}{l}0 \cdot 10 \\
0 \cdot 10 \\
0.08 \\
0.08\end{array}$ & $\begin{array}{l}38 \cdot 8 \\
38 \cdot 9 \\
39 \cdot 2 \\
39 \cdot 6\end{array}$ & 25. IX. " & 1.96 & $\begin{array}{c}8.00 \mathrm{~A}, \mathrm{M}, \\
11.00 \\
2.00 \mathrm{P}, \mathrm{M} . \\
5.00\end{array}$ & $\begin{array}{l}0.13 \\
0.12 \\
0.12 \\
0.11\end{array}$ & $\begin{array}{l}39 \cdot 1 \\
39 \cdot 0 \\
39 \cdot 3\end{array}$ \\
\hline 18. IX. , & 1.88 & $\begin{array}{l}9.00 \text { A.M. } \\
12.00 \\
3.00 \text { P.M. } \\
6.00\end{array}$ & $\begin{array}{l}0.08 \\
0.09 \\
0.08 \\
0.10\end{array}$ & $\begin{array}{l}38 \cdot 7 \\
38 \cdot 8 \\
39 \cdot 4 \\
39 \cdot 9\end{array}$ & 26. IX. " & 200 & $\begin{array}{c}8.00 \text { A.M. } \\
11.00 \\
2.00 \text { P.M. } \\
5.00\end{array}$ & $\begin{array}{l}0.12 \\
0.13 \\
0 \cdot 13 \\
0 \cdot 14\end{array}$ & $\begin{array}{l}39 \cdot 1 \\
39 \cdot 1 \\
39 \cdot 1 \\
39 \cdot 4\end{array}$ \\
\hline 19.1X. " & 1.97 & $\begin{array}{c}8.00 \text { A.M. } \\
11.00 \\
2.00 \text { P.M. } \\
5.00\end{array}$ & $\begin{array}{l}0.11 \\
0 \cdot 12 \\
0 \cdot 13 \\
0 \cdot 12\end{array}$ & $\begin{array}{l}40 \cdot 6 \\
39 \cdot 5 \\
39 \cdot 3 \\
39 \cdot 8\end{array}$ & 27. IX. , & 1.95 & $\begin{array}{l}8.00 \mathrm{A.M} \\
2.00 \mathrm{P.M} \\
5.00\end{array}$ & $\begin{array}{l}0.12 \\
0.12 \\
0.11\end{array}$ & $\begin{array}{l}39 \\
39\end{array}$ \\
\hline $20 . \mathrm{IX}$ & $2 \cdot 10$ & $\begin{array}{c}8.00 \text { A.M. } \\
11.00 \\
2.00 \text { P.M. } \\
5.00\end{array}$ & $\begin{array}{l}0.13 \\
0.14 \\
0 \cdot 15 \\
0.12\end{array}$ & $\begin{array}{l}39 \cdot 7 \\
39 \cdot 4 \\
39 \cdot 3 \\
39 \cdot 4\end{array}$ & 28. IX. , & 1.94 & $\begin{array}{l}9.00 \text { A.M. } \\
1.00 \text { P.M. } \\
5.00\end{array}$ & $\begin{array}{l}0 \cdot 13 \\
0 \cdot 12 \\
0 \cdot 10\end{array}$ & $\begin{array}{l}39 \cdot 0 \\
39 \cdot 0 \\
39 \cdot 3\end{array}$ \\
\hline 22. IX. ", & 1.85 & $\begin{array}{c}9.00 \mathrm{~A}, \mathrm{M} . \\
12.00 \\
3.00 \text { P.M. } \\
6.00\end{array}$ & $\begin{array}{l}0 \cdot 11 \\
0 \cdot 14 \\
0 \cdot 14 \\
0 \cdot 10\end{array}$ & $\begin{array}{l}39 \cdot 1 \\
39 \cdot 3 \\
39 \cdot 7 \\
39 \cdot 4\end{array}$ & & & & & \\
\hline
\end{tabular}

1) A. Th. B. Jacobsen, Biochem. Ztschr., 51 (1913), 448.

2) M. Kageyama. Acta Schola Med. Univers. Imper. Kioto, 1 (1916), 224. (German).

3) 'T. Suzuki, Tokyo-Igakkwai-Zasshi, 30 (1916), 1201. (Jap.)

4) O. Asakawa, Mitteil. med. Fak. Univers., Tokyo, 25 (1921), 529.

5) I. Fujii, this Journ., 2 (1921), 19. 
Rabbit No. 2.

\begin{tabular}{|c|c|c|c|c|c|c|c|c|c|}
\hline$\stackrel{\Xi}{E}$ & 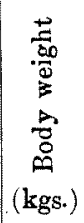 & $\stackrel{\stackrel{\Xi}{\Xi}}{\stackrel{\Xi}{E}}$ & 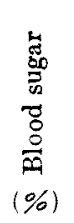 & 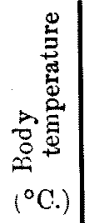 & 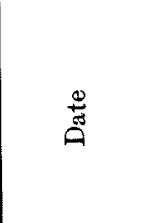 & 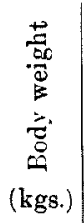 & $\stackrel{0}{\Xi}$ & 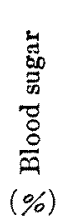 & 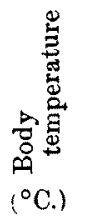 \\
\hline 9. IX.'19 & $1 \cdot 84$ & $\begin{array}{l}11.10 \text { A.M. } \\
2.10 \text { P.M. } \\
4.10\end{array}$ & $\begin{array}{l}0.08 \\
0.08 \\
0.10\end{array}$ & $\begin{array}{l}38 \cdot 9 \\
39 \cdot 2 \\
39 \cdot 7\end{array}$ & 21. IX. '19 & 1.67 & $\begin{array}{l}9.10 \text { A.M. } \\
12.10 \text { P.M. } \\
3.10 \\
6.10\end{array}$ & $\begin{array}{l}0.09 \\
0.13 \\
0.12 \\
0.09\end{array}$ & $\begin{array}{l}38 \cdot 8 \\
38 \cdot 8 \\
39 \cdot 0 \\
39 \cdot 3\end{array}$ \\
\hline 10.IX. , & $1 \cdot 76$ & $\begin{array}{l}8.10 \mathrm{~A} . \mathrm{M} \\
11.10 \\
2.10 \text { P.M. } \\
5.10\end{array}$ & $\begin{array}{l}0.07 \\
007 \\
0.09 \\
0.08\end{array}$ & $\begin{array}{l}38 \cdot 5 \\
38 \cdot 6 \\
38 \cdot 9 \\
39 \cdot 5\end{array}$ & 23. IX. ", & 1.74 & $\begin{array}{c}8.40 \text { A.M. } \\
11.40 \\
2.40 \text { P.M. } \\
5.40\end{array}$ & $\begin{array}{l}0.10 \\
0.11 \\
0.09 \\
009\end{array}$ & $\begin{array}{l}39 \cdot 2 \\
39 \cdot 2 \\
39 \cdot 2 \\
39 \cdot 0\end{array}$ \\
\hline 11. IX. ", & 1.72 & $\begin{array}{c}9.10 \text { A.M. } \\
12.10 \text { P.M. } \\
3.10 \\
9.10 \text { A.M. }\end{array}$ & $\begin{array}{l}0.08 \\
0 \cdot 10 \\
0 \cdot 11 \\
\\
0.08\end{array}$ & $\begin{array}{l}38 \cdot 8 \\
38 \cdot 6 \\
38 \cdot 9 \\
\\
38 \cdot 7\end{array}$ & 24. IX. ", & 1.80 & $\begin{array}{l}8.40 \text { A.M. } \\
11.40 \\
2.40 \text { P.M. } \\
5.40\end{array}$ & $\begin{array}{l}0.09 \\
0.10 \\
0.11 \\
0.10\end{array}$ & $\begin{array}{l}39 \cdot 2 \\
38 \cdot 9 \\
39 \cdot 2 \\
39 \cdot 2\end{array}$ \\
\hline & & $\begin{array}{l}12.10 \text { P.M. } \\
3.10 \\
6.10\end{array}$ & $\begin{array}{l}0.08 \\
0.09 \\
0 \cdot 10\end{array}$ & $\begin{array}{l}38 \cdot 7 \\
39 \cdot 3 \\
39 \cdot 6\end{array}$ & 25. IX. " & $1 \cdot 86$ & $\begin{array}{l}8.10 \text { A.M. } \\
11.10 \\
2.10 \text { P.M. }\end{array}$ & $\begin{array}{l}0.10 \\
0.11 \\
0.12\end{array}$ & $\begin{array}{l}39 \cdot 2 \\
38 \cdot 9 \\
39 \cdot 1\end{array}$ \\
\hline 19.IX. " & $1 \cdot 73$ & $\begin{array}{c}8.10 \text { A.A. } \\
11.10 \\
2.10 \text { P.M. } \\
5.10\end{array}$ & $\begin{array}{l}0.11 \\
0.12 \\
0.13 \\
0.12\end{array}$ & $\begin{array}{l}39 \cdot 6 \\
39 \cdot 2 \\
39 \cdot 3 \\
39 \cdot 8\end{array}$ & 26. IX. " & 1887 & $\begin{array}{r}5.10 \\
8.10 \text { A.M. } \\
11.10 \\
2.10 \text { P.M. }\end{array}$ & $\begin{array}{l}0.12 \\
0.12 \\
0.10 \\
0.12 \\
0.11\end{array}$ & $\begin{array}{l}39 \cdot 1 \\
39 \cdot 1 \\
38 \cdot 9 \\
39 \cdot 9 \\
39 \cdot 2\end{array}$ \\
\hline 20.1X. " & $1 \cdot 75$ & $\begin{array}{r}8.10 \text { A.M. } \\
11.10 \\
2.10 \text { P.M. } \\
5.10\end{array}$ & $\begin{array}{l}0.12 \\
0.13 \\
0.12 \\
0.12\end{array}$ & $\begin{array}{l}39 \cdot 2 \\
39 \cdot 2 \\
39 \cdot 4 \\
39 \cdot 4\end{array}$ & 27. IX. ", & 1.88 & $\begin{array}{l}8.10 \\
8.10 \text { A M. } \\
2.10 \text { P.M. } \\
5.10\end{array}$ & $\begin{array}{l}0.11 \\
0.11 \\
0.11 \\
0.11\end{array}$ & $\begin{array}{l}38 \cdot 8 \\
39 \cdot 1 \\
39 \cdot 3\end{array}$ \\
\hline
\end{tabular}

But naturally, if the direction of the variation of the blood sugar content always agrees with that of the body temperature, though small, it should have some significance.

So, the increase of the blood sugar content was missed in our rabbits in about 30-60 hours after the heat-puncture, contrary to Richter, Senator and Kuno. The body temperature of their rabbits did not exceeded $40 \cdot 7^{\circ} \mathrm{C}$. Senator reported only two protocols from his ten experiments.

\section{Summary.}

Neither hyperglycaemia nor glycosuria was detected in the heatpunctured rabbits whose body temperature rose to $40 \cdot 3-41.5^{\circ} \mathrm{C}$., though the blood sugar content was estimated so long as the body temperature exceeded the physiological limit, i.e. for one half to three days after the puncture. 